\title{
Arbor
}

\section{La Cooperación Internacional entre Servicios}

\author{
Julia Pulido Gragera
}

Arbor CLXXX, 709 (Enero 2005), 269-288 pp.

Los nuevos requerimientos en materia de seguridad que la comunidad internacional tiene que asumir como consecuencia de las nuevas amenazas y riesgos, obligan a que los Servicios de Inteligencia y de Información, objeto de análisis de este artículo, incrementen y mejoren la colaboración $y$ cooperación entre ellos.

En este sentido la aspiración de los Estados por poseer el máximo nivel de seguridad cediendo el mínimo de soberanía, se constituye como uno de los objetivos principales de los mismos, dificultad que se presenta, en la actualidad, para poder lograr una efectiva coordinación multilateral. De esta forma, no solamente se tendrá que tener en cuenta las necesidades vitales en cuestiones de Inteligencia, que obligan a los Estados a establecer relaciones de cooperación multilaterales, sino que se deberá analizar la posición que defiende cada uno de ellos como actor y como sujeto de soberanía en las Relaciones Internacionales.

Para ello, el análisis de los diferentes niveles de cooperación existentes en materia de Inteligencia, intraestatal e interestatal, junto con las iniciativas de colaboración e intercambio de información que se han llevado a cabo en el panorama internacional, y especialmente en el ámbito Europeo, serán los pilares en los que se sustentará este artículo.

\section{Introducción}

A riesgo de simplificar, podría considerarse que los Servicios de Inteligencia son un reflejo de la idiosincrasia de un Estado. A lo largo de los 
siglos, la obtención de información se ha considerado un objetivo esencial para lograr una óptima seguridad y defensa. La necesidad de prever acciones ofensivas y el interés por el conocimiento de las realidades del sistema internacional, son factores que han impulsado a los Estados a utilizar diferentes métodos para la obtención de información.

En la actualidad, el aumento de actores en el sistema internacional que influyen en la realidad estatal, provoca que se necesite un conocimiento tanto de sus propósitos como de sus actuaciones.

Por ello, los Servicios de Inteligencia e Información se constituyen en objeto de análisis desde dos perspectivas. Por un lado, como entidades propias con carácter de Administración del Estado, y por otro como instrumentos de estos.

En este caso, la dimensión del análisis será la última, puesto que el enfoque estará centrado en la cooperación internacional de los Estados a través de sus Servicios de Inteligencia.

En un sentido general, el cambio en el concepto de seguridad que tras el final de la Guerra Fría ${ }^{1}$ ha supuesto una reestructuración de las funciones y los objetivos de los Servicios de Inteligencia, ha requerido el establecimiento de unos mecanismos de cooperación entre los mismos no exentos de dificultades.

La necesidad vital de los Estados de obtener la máxima seguridad posible ante un amplio espectro de amenazas, obliga a que incurran en el establecimiento de mecanismos preventivos o reactivos para contrarrestarlas. En este sentido, y en lo que corresponde a las medidas de prevención, la labor tanto de los Servicios de Inteligencia como de Información es fundamental en la consecución de dichos objetivos.

Un dato a tener en cuenta, es la dificultad que los Estados, como actores del Sistema Internacional, presentan en la convivencia dentro del mismo.

El deseo de los países en la consecución de la máxima eficacia en la provisión de seguridad a través de las organizaciones internacionales se ve, paradójicamente, contrapuesta con las restricciones a la hora de compartir Inteligencia por motivos de salvaguarda de los intereses nacionales propios de los países.

\footnotetext{
1 Ya el profesor Barry Buzan establecía en 1991 un concepto ampliado de seguridad que progresivamente ha ido evolucionando durante la última década. En este sentido véase B. BuzAn, People, States and Fear, Hemel Hemstead, Harvester Whatsheaf, 1991 (2. ${ }^{\mathrm{a}}$ ed.).
} 


\section{La Cooperación Internacional entre Servicios}

En este sentido, la pretensión de alcanzar seguridad implica un intento de equilibrio a través de la integración de los Estados en las organizaciones internacionales, siendo paradójicamente, reacios a considerar que la seguridad del otro puede llegar a ser la seguridad propia. De esta forma se materializa la tesis de Berki², considerando que la seguridad no es un concepto absoluto. La situación «ideal» en estos términos no existe, considerándose el factor individual como elemento fundamental a la hora de valorar el concepto de la misma, ya que ningún sujeto llega a alcanzar la seguridad completa por sí y para sí mismo. Depende de lo que la colectividad llegue a conseguir para la suya propia ${ }^{3}$.

Trasladándose esta teoría a la práctica interestatal, la aspiración a la utópica seguridad global absoluta que puede detentar un Estado, se convierte en la carencia de ésta por parte del resto. Se puede decir, por tanto, que la seguridad propia es la inseguridad del contrario. Al ser éste un concepto relativo, la seguridad es susceptible de presentar vulnerabilidades que pueden transformarse en la distinta percepción de riesgos y amenazas que pueden poseer Estados distintos.

La necesidad de lograr una seguridad lo más efectiva posible por parte de los Estados es algo indiscutible. La cuestión radica en determinar la vía para conseguirla.

En este sentido, las posibilidades de cooperación entre distintos Estados para la obtención de un mayor grado de seguridad, se ven mermadas de dos formas distintas. Por una parte, es innegable que la cooperación puede aportar ventajas para todas las partes, pero lo hace en distinta medida. Algunos autores consideran que las ventajas obtenidas no son absolutas, sino relativas. Cuando dos Estados se disponen a cooperar en una ventaja mutua la pregunta que en un primer momento surge es: si no ganamos los dos por igual, ¿quién ganará más? «El hecho de que un Estado se vea más beneficiado en términos proporcionales, puede traducirse en el incremento de sus capacidades, y por tanto, en la aparición de una amenaza para el resto» ${ }^{4}$.

2 R. N. BERKI, Security and Society: Reflections.on Law, Order and Politics, London, 1986, pp. 28-43.

3 En este sentido, la obra de referencia sobre la creación de una comunidad de seguridad es el clásico de Karl Deustcht, Political Community in the North Atlantic Area. Princeton, 1957. También se puede consultar la obra de Robert JERVIS «Security Regimes» en el libro de S. KRASNER, (ed.) International Regimes. Ithaca, NY. Cornell University Press, 1993.

4 K. SoduPE, La Teoría de las Relaciones Internacionales a comienzos del siglo XXI. op. cit., p. 89. 
La segunda forma está ligada con la primera, ya que se puede decir que una mayor interdependencia entre distintos Estados produce vulnerabilidades puesto que las grandes potencias tienden a controlar aquello de lo que dependen o disminuir el grado de dependencia. Por tanto, el potencial para la cooperación entre Estados sería un producto de intereses y preferencias. De hecho, hay conductas que pueden elegir la cooperación porque otra decisión puede llevar a resultados no deseados o sub-óptimos 5 .

En cuanto al objetivo principal de los Estados, su seguridad, para algunos autores, los Estados forman alianzas no solo para equilibrar sus fuerzas sino para contrarrestar cualquier indicio de amenaza para su independencia ${ }^{6}$.

En síntesis se puede decir que cuanto más recursos de poder tenga un Estado, mayor potencialidad posee para convertirse en amenaza para el resto. Por tanto, la variable "amenaza» es junto con la distribución de poderes, el factor que incide de una manera directa en la formación de alianzas. En este sentido, se puede afirmar que en el factor "amenaza" se incluye tres variables ${ }^{7}$ : la proximidad geográfica, por la que los Estados muestran más propensión a formar alianzas para contrarrestar amenazas cercanas. Los recursos ofensivos por los que un Estado al aumentar su potencial militar puede suponer un peligro para la integridad territorial y soberanía de terceros. En este sentido, los Estados que perciban encontrarse en inferioridad de capacidades con respecto al primero, recurrirán a la formación de alianzas para contrarrestar su capacidad ofensiva.

Finalmente, la tercera variable, se determina por las intenciones de los Estados, en tanto en cuanto, las percepciones de los terceros son esenciales para la consecución de una alianza en contra del primero, es decir, aquellos Estados que son percibidos como especialmente peligrosos provocan que se formen coaliciones contra ellos para equilibrar el potencial peligro.

Pero en la actualidad la consideración de amenaza o riesgo a la seguridad no se circunscribe exclusivamente al ámbito estatal, el surgimiento de una nueva concepción de los mismos, así como la ampliación de lo que se considera espectro del conflicto, obliga a reforzar los lazos de cooperación entre los Servicios de Inteligencia e Información para poder dar respuesta a los nuevos retos, aunque para ello hay que vencer las dificultades intrínsecas al concepto de cooperación.

5 El ejemplo clásico se define a través del dilema del prisionero.

6 S. M. WALT, "Alliances Threats and U.S. Grand Strategy: A Reply to Kaufman and Labs", Security Studies. Vol.1, n 3 , (1992), p. 450.

7 Ibídem. 


\section{La Cooperación Internacional entre Servicios}

\section{La Cooperación Internacional entre Servicios de Inteligencia}

En la actualidad, amenazas como el surgimiento de proliferación de armas de destrucción masiva, conflictos intraestatales, el terrorismo y su interconexión con el crimen organizado, etc., suponen unos retos a los que la sociedad internacional debe responder de una forma preventiva y reactiva.

Así, desde la postura estatal que, a través del responsable político marca las directrices de los Servicios de Inteligencia, la labor preventiva de los mismos debe inscribirse en un ámbito multidimensional en el que la cooperación internacional se aprecia como fundamental, aunque no exenta de matizaciones.

Desde una perspectiva del estudio de los Servicios de Inteligencia y de Información como instrumentos de los Estados para la realización de políticas preventivas, la cooperación entre ellos se presenta con importantes dificultades en cuanto a la eficacia de la misma.

Si se considera que el Estado es el actor principal en las Relaciones Internacionales, los Servicios de Inteligencia y de Información que actúan al servicio de los mismos, contarán con las mismas dificultades que los países a los que representan a la hora de establecer relaciones de cooperación. De esta forma, los Servicios de Inteligencia van a actuar conforme a sus propios intereses y anteponiendo la Seguridad Nacional del Estado al que pertenecen, a las necesidades que, de una forma colectiva, se tomen en una coalición.

Por ello, la clave para lograr un entendimiento se encuentra en la búsqueda de objetivos comunes y en el que se logre un equilibrio de poder entre los actores, en el que ninguno se vea en una posición desproporcionada por un incremento de beneficios para un actor en detrimento de los otros, en este caso, de los Servicios de Inteligencia.

En este sentido, la consecución de un entendimiento con respecto a unas materias determinadas que representan una amenaza real para los Estados se establece como el primer paso para una cooperación efectiva. Actualmente, una de estas materias es el terrorismo, pero esto no es suficiente si no hay un acuerdo sobre los mecanismos preventivos, de los cuáles forman parte los Servicios de Inteligencia, como elemento esencial.

El problema surge a la hora de necesitar compartir cierta información considerada por un Estado que puede afectar a su propia Seguridad Nacional. Este caso es un claro ejemplo de aseguración de la supervivencia de un Estado, en el que la protección celosa de la información puede suponer convertirse en un factor negativo en la eficacia de la cooperación internacional. Pero indiscutiblemente es un cuestión inevitable. 
En lo referente al hecho que hacen que los Estados establezcan un equilibrio de poder, la aplicación a los Servicios de Inteligencia en la actualidad es acertada en tanto en cuanto, el objetivo principal es la supervivencia dentro del sistema. Cada Servicio de Inteligencia, como ya se ha apuntado, tiene el deber principal de salvaguardar los intereses nacionales y la seguridad nacional frente a amenazas, internas o externas.

Asimismo, la necesidad por parte de los Estados de minimizar los efectos que derivados de los "dilemas de seguridad», obligan a anhelar la posesión del control de los riesgos y amenazas por parte de los Servicios de Inteligencia, dominando situaciones adversas que no se caracterizan por ser los propios Estados las productoras de las mismas, como en muchas ocasiones es el terrorismo.

Finalmente, la cuestión de las percepciones es algo fundamental porque de ellas va a depender que se tomen los requerimientos y las decisiones oportunas en un determinado momento, no solamente al más alto nivel, como es el caso del responsable político, sino a todos los niveles dentro del propio Servicio establecidos en el Ciclo de Inteligencia ${ }^{8}$.

\subsection{Niveles de cooperación entre los Servicios de Inteligencia e Información}

Se ha mencionado que el aspecto fundamental en la actualidad para lograr una óptima cooperación entre Servicios es el establecimiento de una estrategia en la que se establezcan materias y objetivos comunes.

En este sentido, se puede decir que existen dos grandes niveles: el intraestatal, basado en la cooperación entre los distintos Servicios de Inteligencia e Información que operan en un Estado, y el nivel interestatal, que, a su vez, se divide en cooperación bilateral y multilateral.

\section{a) Nivel Intraestatal}

En cuanto a este nivel, las dificultades que se presentan en la cooperación entre los distintos Servicios surgen por dos motivos principalmente. Por un lado, a consecuencia de la excesiva burocratización en los me-

8 B. Berkowitz y A. E. Goodman, Strategic Intelligence for American National Security, Princeton, New Jersey, Princeton University Press, 1989, p. 33. 


\section{La Cooperación Internacional entre Servicios}

canismos de intercambio de información. Y por otro, por la poco exacta demarcación de las competencias asignadas a cada uno de los Servicios, por lo que, algunos de ellos ejecuta funciones que no le corresponden, dificultando y duplicando las tareas con la consiguiente demora en los objetivos encomendados.

En lo correspondiente a los Servicios de Inteligencia y de Información policiales, tradicionalmente han actuado en esferas diferentes. Los primeros han mantenido su ámbito de actuación enfocado en los planes y capacidades de gobiernos extranjeros, mientras que los segundos se han centrado en neutralizar la actividad criminal dentro de las fronteras de los Estados.

Actualmente, los actos incardinados en el paraguas del crimen organizado llegan a presentar una naturaleza global, por lo que no llegan a ser competencia exclusiva de las Fuerzas de Seguridad y de sus Servicios de información, sino que la obtención y el análisis de la información sobre estos asuntos llegan a ser prioridad de la Comunidad de Inteligencia en su conjunto.

Pero, las dificultades se presentan a la hora de establecer las misiones, los objetivos y los requerimientos legales, sobre todo en aquellos Estados en los que las competencias de ambos Servicios, no están claramente delimitadas.

En este sentido, se puede decir que, en general, hay dos fuentes principales de conflicto en el nivel de cooperación intraestatal. Por un lado, la re ticencia a la hora de compartir información sensible. Algunos Servicios de Información se reservan los datos relativos a terrorismo, crimen organiza do, etc., obtenidos en el transcurso de una investigación criminal, por lo que dificulta el vínculo de comunicación entre otras Agencias de Inteligencia.

La segunda fuente de conflicto se refiere a que algunos Servicios de Información han incrementado sus esfuerzos en ampliar sus ámbitos de actuación, invadiendo, en cierta forma, las áreas competenciales de los Servicios de Inteligencia. Así, en Estados Unidos, el FBI, en ciertos aspectos, emprende investigaciones de forma conjunta con otros Servicios de Información homólogos extranjeros y desarrolla fuentes de información independientes sobre actividades relacionadas con el crimen organizado. Este hecho provoca algunas situaciones de ingerencia en competencias enfocadas al exterior propias de las Agencias de Inteligencia que, en algunos casos, podría ser beneficioso para las mismas, si se estableciera un vínculo de coordinación efectivo que pudiera facilitar el cumplimiento de las tareas de las Agencias de Inteligencia a través de la experiencia de los Servicios de Información en el ámbito de las actividades criminales. Pero, ciertamente, el hecho de que cada Servicio quiera guar- 
dar celosamente la información que han obtenido, complica en exceso la voluntad de establecer unos canales fluidos de intercambio de datos.

Como ejemplo clarificador, se puede exponer el ocurrido tras el 11-S entre las Agencias de Inteligencia e Información en Estados Unidos, en cuanto a los fallos detectados.

Se puede decir que el 11-S se basó principalmente en el vacío en la agilidad entre las distintas Agencias, como consecuencia de la falta de directrices políticas precisas conforme a las necesidades de Inteligencia.

A pesar de las iniciativas establecidas ya desde a década de los noventa, como el establecimiento de un grupo de trabajo conjunto entre las Agencias de Seguridad y los Servicios de Inteligencia en 1995 compuesto por expertos en jurisprudencia y otros oficiales pertenecientes a las mismas, y la formación de un grupo de coordinación con el objetivo de establecer las divisiones competenciales adecuadas y los mecanismos para la resolución de diferencias entre Servicios que puedan surgir, los problemas en la coordinación entre Agencias culminaron en la poca previsión y escasa agilidad de la que hicieron gala los Servicios de Inteligencia estadounidenses en los atentados terroristas del 11-S.

Por tanto, la falta de cooperación entre las Agencias de Inteligencia y las de información, concretamente entre la CIA y el FBI, ha sido motivo de discusión considerándose una de las posibles causas de la falta de previsión de los atentados. Por ello, se han establecido una serie de mecanismos de colaboración más profunda, así como una reforma de la $\mathrm{Co}$ munidad de Inteligencia en su conjunto.

La división de competencias entre las agencias de inteligencia y las policiales estadounidenses habían estado especificadas desde hace décadas. La Comunidad de Inteligencia se centraban en las amenazas militares que provenían del exterior, mientras que el terrorismo competía a las agencias policiales, concretamente al FBI (Federal Bureau of Investigation). Desde el final de la Guerra Fría, a comienzos de los noventa, se comenzaron a dar pasos para establecer una estrecha coordinación entre las dos comunidades. Esto se materializó en el USA Patriot Act, como resultado de los atentados producidos en septiembre del $2001^{9}$.

La separación que a lo largo de la historia han mantenido los sucesivos gobiernos estadounidenses, de los asuntos que correspondían a lo relacionado con las actividades criminales y las que eran concernientes a la

9 R. BEST, «Intelligence to Counter Terrorism: Issues for Congress». CRS Report for Congress. (Febrero 2002). 


\section{La Cooperación Internacional entre Servicios}

Seguridad Nacional se puso de manifiesto en el FISA (Foreign Intelligence Surveillance Act). Establecido en 1978 establece un sistema por el que se autoriza el seguimiento y la obtención de información relativa a la Seguridad Nacional. Los procedimientos difieren dependiendo de que tipo de amenaza o riesgo se refiera. Tras los atentados, se introduce una serie de modificaciones recogidas en el USA Patriot Act. Básicamente, se pretendió establecer una mejor coordinación entre CIA y FBI a la hora de compartir información en tiempo real.

Uno de los obstáculos que se aprecia para poder establecer una coordinación y unas labores conjuntas entre CIA y FBI es el impedimento burocrático en cuanto a las posibles modificaciones de las competencias de cada una de las Agencias. Las funciones y competencias están recogidas en estatutos diferentes y la regulación en la clasificación de la información es extremadamente rigurosa. Por lo tanto, la dificultad a la hora de compartir cierta información entre las mismas tiene que ser autorizada burocráticamente. De esta forma no solamente se demanda una reestructuración de los diferentes Servicios en sus funciones y actuaciones, sino que se plantea unas medidas estrictas en el control de los mismos.

El Congreso mediante diferentes comités tiene la labor de someter a las agencias de Inteligencia e información a una revisión y a un control tanto de sus presupuestos como de las actividades. En este sentido también se aprecian diferencias entre los distintos órganos que ejercen el control de las agencias de inteligencia y las policiales. La Oficina de Gestión y del Presupuesto (Office of Management and Budget) establece anualmente el presupuesto repartidos por partidas para cada una de las agencias.

En la actualidad, la aprobación del proyecto de ley de reforma de la Inteligencia supone un paso adelante, no solo en la reestructuración de las Agencias en cuanto a funciones y objetivos, sino en el establecimiento de mecanismos de cooperación más eficaces entre ellas.

Por tanto, si el problema de las organizaciones terroristas actuales es su poder y capacidad para poder atacar en cualquier dirección y lugar con una gran variedad de métodos no convencionales, se tiene que estar en disposición de poder obtener información de una gran variedad de fuentes y establecer el análisis oportuno en el más breve espacio de tiempo. Para ello se debe contar con un número de expertos que puedan establecer el nivel de alerta necesario para que el responsable político pueda tomar las medidas oportunas.

Por tanto, se requiere un nuevo objetivo centrado en el establecer organizaciones de Inteligencia ágiles que tengan habilidad para adaptarse 
a los cambios. Asimismo, tienen que contar con un conjunto de individuos, fuentes de información y metodología que resuelva una situación en la actualidad y que pueda ser modificada de una forma ágil cuando las amenazas cambien.

El concepto de «agilidad», por tanto, es una idea a considerar en los círculos de inteligencia. En este sentido, Berkowitz establece cuatro factores necesarios para que se produzca la «agilidad» necesaria. El primero es que la organización necesita ser capaz de cambiar y modificar al personal y a otros recursos lo más rápida y eficientemente posible cuando sea requerido por las situaciones.

En segundo lugar, la necesidad de ser capaz de disponer expertos y fuentes de información en todo el mundo.

Tercero, poseer la capacidad de proporcionar la información obtenida lo más rápido posible para que se puedan realizar los análisis de la misma en el más breve plazo de tiempo y de una forma efectiva.

Finalmente, el facilitar la Inteligencia a los consumidores de la forma que sea requerida para cada trabajo ${ }^{10}$.

Un ejemplo de cooperación intraestatal es el desarrollado en nuestro país con la creación del Centro Nacional de Coordinación Antiterrorista (CNCA). Aprobado por Consejo de Ministros el 28 de mayo del 2004, tiene el objetivo principal de integrar la información estratégica relativa al terrorismo, tanto de proyección nacional como internacional. La naturaleza del mismo es de carácter complementario y auxiliar de la Fuerzas de Seguridad del Estado, no asumiendo poderes ejecutivos coercitivos, los cuáles formaran parte exclusivamente de los Cuerpos de Seguridad del Estado. Este Centro actúa como órgano de recepción, proceso y valoración de la información estratégica disponible sobre terrorismo que constituye una amenaza para España, integrando, valorando y analizando toda la información disponible. La estructura de personal se caracteriza por disponer de miembros de los Cuerpos y Fuerzas de Seguridad del Estado ( Cuerpo Nacional de Policía y Guardia Civil) y un número determinado de funcionarios que el Ministerio de Defensa considere oportuno procedentes del Centro Nacional de Inteligencia ${ }^{11}$.

10 B. BeRKowiTZ, «Spying in the Post- September 11 World». http://www-hover.stanford.edu/publications/digest/034/berkowitz.html.

11 Véase a este respecto el Acuerdo del Consejo de Ministros del 28 de mayo de 2004, por el que se crea el CNCA y la Orden INT/1251/2004, del 7 de mayo, por la que se crea el Comité Ejecutivo para el Mando Unificado de las Fuerzas y Cuerpos de Seguridad del Estado. 


\section{La Cooperación Internacional entre Servicios}

\section{b) Nivel Interestatal}

En este sentido, las dificultades que se plantean no son tanto desde el punto de vista burocrático y de competencias, como del establecimiento de una cooperación en materias comunes junto con un fluido intercambio de Inteligencia entre distintos Servicios.

En este sentido se puede destacar dos niveles de cooperación: el bilateral y el multilateral.

Desde el punto de vista bilateral se puede decir que es el nivel más común de cooperación entre Servicios de Inteligencia. Se puede realizar de una manera formal, a través de la firma de Memoranda de Entendimiento (MoU's) ${ }^{12}$ o acuerdos expresos por parte de los Estados por los que se garantiza la confidencialidad de la información, o de un modo informal, mediante acuerdos tácitos, entre distintos Servicios de Inteligencia en determinadas materias.

En este caso, la práctica de la cooperación resulta más sencilla estableciéndose un intercambio de información desde un equilibrio de poder, en el que los Estados «intercambian cromos» desde una situación de igual a igual y asuntos de interés mutuo. Cada Estado a través de sus Servicios de Inteligencia establece enlaces en los Estados que le resulten de cierto interés para la consecución de sus propósitos políticos nacionales o que sean relevantes para el mantenimiento de su acción exterior o intereses nacionales. Un ejemplo de cooperación es la desempeñada por la Organización de Inteligencia de Seguridad Australiana (ASIO) y por el Servicio de Inteligencia de Seguridad Canadiense (CSIS). El primero estableció en el 2003 doscientos treinta y tres enlaces, distribuidos en ciento cuatro Estados. En cuanto al segundo, presenta ofíciales de enlace permanentes establecidos a lo largo de la geografía mundial ${ }^{13}$.

La tipología de niveles de cooperación bilateral se establece atendiendo a diversos tipos de acuerdos. En este sentido, se pueden firmar acuerdos por los que un Estado comparte la obtención o el análisis de la información con otro en un área determinada, con la consiguiente reciprocidad por parte del segundo en otro ámbito.

Otro tipo de cooperación es el que se realiza cuando un Estado permite a otro obtener información en su territorio a cambio de que comparta con el primero el resultado de esa obtención.

12 Son las siglas de «Memorandum of Understanding».

13 S. LefebVRE, «The Difficulties and Dilemmas of International Intelligence Cooperation", International Journal of Intelligence and Counter intelligence, $\mathrm{n}^{\circ} 16$ (2003), p. 533. 
En tercer lugar, un país puede ayudar a otro a adquirir cierta información para sus propios intereses con la condición de que el primero pueda beneficiarse con el intercambio de los resultados.

En cuarto lugar, se pueden establecer operaciones conjuntas para la obtención de información entre dos Estados que compartan el mismo interés en un ámbito o un asunto determinado.

Finalmente, otro tipo de acuerdo es el que se establece para intercambiar analistas y técnicos entre dos países, así como proporcionar formación a personal de un Servicio extranjero a cambio de las ayudas prestadas en un determinado momento por otro país. En este sentido, se puede destacar, por ejemplo, la asistencia en la provisión de traductores a un Servicio por parte de otro en un área geográfica determinada.

Hay que decir que en numerosas ocasiones se produce una confusión entre los conceptos de cooperación bilateral desigual y cooperación asimé trica. Normalmente, es complicado que se produzca una simetría en los intercambios de información entre dos Estados, por la razón de que las necesidades de inteligencia y las capacidades para obtener la información de un Servicio no tienen por que coincidir plenamente con las del Estado con el que mantienen el acuerdo de cooperación bilateral. En cambio, se produce una colaboración desigual cuando hay una brecha sustancial cuantitativa y cualitativamente en el intercambio de información.

En síntesis, la base para una eficaz cooperación bilateral se resume en la consideración por parte de los Estados de que el elemento fundamental para una eficaz cooperación es la reciprocidad en el intercambio de Inteligencia y/o información, y no tanto la posición de igualdad de los Estados en cuanto a ese intercambio puesto que siempre se va a establecer un equilibrio entre las dos voluntades políticas. Con ello, se quiere afirmar que las ventajas van a ser relativas y no siempre absolutas puesto que la valoración que se realiza de los intereses se efectúa a través del juego de las percepciones siendo una elección más cualitativa que cuantitativa.

En cuanto al nivel multilateral, es necesario destacar la dificultad que plantea el establecimiento de una relación de cooperación eficaz, por las razones mencionadas al comienzo del capítulo. Cada Estado presenta unas necesidades conforme a su Seguridad e Interés Nacional, que debe salvaguardar. Este hecho provoca que sean reticentes a compartir parcelas de información que pueden afectar de una forma directa o indirecta al Estado en el desarrollo de sus políticas.

Dentro de este tipo de cooperación, las dos variables que facilitan que ésta se produzca de una forma efectiva y eficiente son, por un lado, la consecución de unos intereses y materias comunes, y por otro, el factor regional. 


\section{La Cooperación Internacional entre Servicios}

En lo que respecta a la primera variable, los Estados que comparten intereses comunes en materias que se constituyen como amenazas a la Seguridad han demostrado su propensión a establecer redes formales para la cooperación en Inteligencia. En raras ocasiones, estas redes que se constituyen como "grupos» o "clubs» van más allá del simple intercambio de información.

Hay que decir, en cuanto organizaciones internacionales como la OTAN o Naciones Unidas, que no poseen capacidades orgánicas propias de Inteligencia, por lo que los requerimientos que se efectúen en este sentido dependerán del grado de información que quieran compartir los Estados miembros.

A partir del 11-S, la toma de conciencia de la incapacidad para hacer frente a la amenaza terrorista de forma exclusivamente estatal, promueve una línea de actuación, por la que se constituyen una serie de alianzas contra el peligro que supone el terrorismo para la seguridad. Pero el establecimiento de este tipo de coaliciones no es nuevo.

Si analizamos las alianzas de Estados establecidas en las últimas décadas en el ámbito de la Inteligencia, nos damos cuenta que se establecen para un fin concreto. Así, el programa UKUSA, antecesor de la red ECHELON, nació de un acuerdo bilateral anglo-americano durante la Segunda Guerra Mundial de cooperación en materia de SIGINT para controlar las señales emitidas por Alemania y Japón. Tras la Guerra, se acordó seguir manteniendo este acuerdo como forma de continuar la cooperación en tiempo de paz. Años más tarde se hizo extensivo a Canadá y a Australia, ampliándose a Nueva Zelanda, con la consiguiente formación de la red ECHELON con el fin de conducir las actividades de Inteligencia de comunicaciones (COMINT) de una forma global.

Complementando a la anterior, la creación en la década de los sesenta del Club CAZAB, fomentado por el entonces Director de la CIA James Angleton, supuso un importante paso en la promoción del intercambio de información entre los Estados pertenecientes a UKUSA en el desarrollo de la contrainteligencia contra la Unión Soviética. Los mecanismos de cooperación se establecían en reuniones anuales entre los responsables de los Servicios de Inteligencia y Contrainteligencia de los Estados miembros, con el objetivo de facilitar la cooperación y el intercambio de información, paralelamente a los fines de colaboración de SIGINT que UKUSA detentaba ${ }^{14}$.

${ }^{14}$ M. RUDNER, «Hunters and Gatherers: The Intelligence Coalition Against Islamic Terrorism». International Journal of Intelligence and Counterintelligence, 17 (2004), pp. 193-230. 
Incluso esta alianza realizada entre Estados con unos intereses comunes y socios con una tradición de colaboración política y económica estrecha, presenta una doble vulnerabilidad en la cooperación. Por una parte, para la urgencia que pueden poseer algunos Estados socios en los requerimientos de su Seguridad Nacional. Y por otro, para las prescripciones y obligaciones políticas y legales de estos. La consecuencia de ello es que los acuerdos informales de la red de trabajo se establecieron como urgentes desde el plano operacional, pero con una contrapartida de carácter muy sensible en lo político, y para las Fuerzas de Seguridad y Servicios de Información policiales de los Estados miembros, que tenían que evaluar si la urgencia de los requerimientos de sus socios, no se contraponían a lo establecido en la normativa legal nacional.

Dentro de las organizaciones internacionales, el Comité Especial de la OTAN, a pesar de no poseer una especial relevancia en cuanto a capacidades propias de obtención de información, debe tener una especial mención en este capítulo por ser instituido por la Alianza Atlántica con el cometido de establecer una estrecha cooperación en el intercambio de Inteligencia Estratégica entre los Servicios de Inteligencia de los Estados miembros. Tras los atentados del 11-S, este Comité creó una unidad analítica para proporcionar al Consejo de la Alianza informes regulares de las amenazas que pudieran poner en peligro la Seguridad de la OTAN, así como la de los Estados miembros, basados en la Inteligencia recibida de los Servicios de Inteligencia de los países pertenecientes a la Organización. Además, este Comité también consideró de importancia el intensificar las relaciones de cooperación con Rusia en el ámbito de la lucha contra el terrorismo, contando, asimismo, con la participación de los Estados pertenecientes al Consejo de Asociación Euroatlántica, como parte del Plan de Acción 2000-2002 ${ }^{15}$.

Entre otros acuerdos multilaterales hay que destacar el Club de Berna, creado en 1971, como grupo de cooperación de carácter multilateral. En él se reúnen anualmente los directores de Inteligencia de los distintos Servicios europeos con el objetivo de establecer la agenda referente a la cooperación entre los diferentes Estados a partir de los intereses comunes concernientes a la Seguridad europea. A partir de 1999, se establece en la agenda las cuestiones relativas a terrorismo, interceptación de comunicaciones, encriptación, y ciberterrorismo, y en el 2000 el Club incluye las cuestiones relativas al papel de los Servicios en la integración europea.

15 Ibídem. 
El Club mantiene su propio sistema de comunicación para distribuir los informes realizados así como la información solicitada. Tras el 11-S, el Consejo europeo sobre Asuntos de Justicia e Interior estableció una serie de tareas al Club con el fin de proporcionar unas líneas de acción a Europol en el ámbito del contraterrorismo. En este sentido, se ha establecido un grupo asesor formado por los directores de unidades contraterroristas, los cuáles, en reuniones periódicas proporcionan asesoramiento a Europol en la lucha contra éste.

El Grupo TREVI (Terrorism, Radicalism, Extrermism and International Violence) fue establecido en el Consejo europeo de Roma de diciembre de 1975, con la misión de intercambiar información y coordinar los esfuerzos en la lucha contra el terrorismo. La creación de este grupo se debió a la preocupación que existía en Europa por la ola de atentados que se estaban produciendo. Grupos terroristas de carácter independentista que actuaban en países como España, Irlanda, y revolucionarios extremistas cuyos objetivos eran Francia, Alemania, Bélgica o Italia, eran objeto de preocupación para los Estados europeos. En este sentido, se crea el grupo con el fin de establecer acuerdos de asesoramiento e intercambios de información.

El Grupo Kilowatt, creado en 1977, surge tras el aumento del terrorismo de corte islamista. Los Estados que forman parte del mismo son Bélgica, Francia, Alemania, Irlanda, Italia, Luxemburgo, Países Bajos, Noruega, Reino Unido, Sudáfrica, Canadá, Noruega, Suecia, Suiza, Estados Unidos e Israel. El objetivo para la creación de este Grupo fue el compromiso de cooperación en el intercambio de información para hacer frente a la dimensión internacional de la amenaza. Actualmente, se han incluido los nuevos Estados que han entrado a formar parte de la Unión Europea.

Paralelamente, al Grupo Kilowatt, se creó el grupo MEGATON, con el objetivo de afrontar las amenazas terroristas de carácter no islámico, como las de corte anárquico y radical.

La diferencia entre estos dos grupos y el resto de coaliciones de cooperación multilateral, radica en que el intercambio de inteligencia no está establecido desde una base de reciprocidad por lo que se aumentan las capacidades contraterroristas de cada Estado y del grupo en su conjunto.

Dentro de las amenazas que ponen en peligro la Seguridad Internacional, no hay que olvidar que no sólo el terrorismo, y la proliferación de armas de destrucción masiva, son consideradas como tales, el crimen organizado, y fundamentalmente lo relacionado con delitos económicos como el lavado de dinero y la financiación de grupos terroristas, tienen 
que tener un tratamiento especial. Por ello, la creación de unidades de Inteligencia financiera como el Grupo Egmont, son imprescindibles como complemento a los grupos de coordinación anteriormente mencionados.

El Grupo Egmont se establece a partir de 1995 como resultado de la unión informal de varias unidades de Inteligencia financiera que comenzaron a operar a principios de los noventa. El principal objetivo de este grupo fue el proporcionar un foro en donde las unidades pudieran obtener apoyo para sus respectivos programas nacionales de lucha contra el blanqueo de dinero. El apoyo incluye la sistematización del intercambio de Inteligencia financiera, proporcionando expertos y capacidades suficientes para cada organización y reforzando la comunicación entre las diferentes unidades a través de la aplicación de las nuevas tecnologías.

Las condiciones para el intercambio de información entre las unidades se establece de una forma libre desde la reciprocidad o el acuerdo mutuo. La información tiene que ser utilizada para el requerimiento del que ha sido objeto, con propósitos específicos. Asimismo, toda la información debe estar sujeta a estrictos controles y salvaguardas para garantizar que el uso de la misma se realiza exclusivamente de una forma autorizada, a través de procedimientos de protección de datos. En la actualidad el Grupo Egmont está compuesto por ochenta y cuatro Estados ${ }^{16}$.

Dentro de la cooperación multilateral, no se puede olvidar el papel que representa la Unión Europea en este sentido. Se puede decir que las motivaciones que presenta la Unión para establecer unos vínculos sólidos en materia de cooperación en Inteligencia, se pueden considerar como una unión entre la variable «materia-interés común», y entre el factor regional.

Es evidente, como se ha puesto de manifiesto, que la cooperación en el ámbito de la Inteligencia resulta complicada como consecuencia de múltiples variables. En la Unión el establecimiento de órganos que faciliten el intercambio de información a nivel comunitario es una pretensión a lograr desde el Tratado de Maastricht.

Tras los atentados del 11-S, y concretamente después de los cometidos en Madrid el 11 de marzo de 2004, la Unión Europea comienza a plantearse la necesidad de un redimensionamiento de los Servicios de Inteligencia y de información, pero desde una perspectiva de definición de objetivos.

16 «The Egmont Group of Financial Intelligence Units», URL: http://www.oecd.org/ fatf/ctry-orgpages/org-egmont_en.htm. 


\section{La Cooperación Internacional entre Servicios}

Europa se da cuenta de las vulnerabilidades que posee intrínsecas a su política comunitaria. La libertad de movimientos proporciona una ventaja a los grupos terroristas y a los individuos que los forman para poder desplazarse sin problemas entre los países del Acuerdo Schengen. Asimismo los terroristas se benefician de las leyes en cuanto al asilo e inmigración que se han establecido en la Unión, así como de las libertades civiles de asociación, religión y lengua.

Por tanto, en los últimos años, y a tenor de los acontecimientos, se ha puesto de manifiesto el anhelo de establecer una eficaz y eficiente política europea de inteligencia. La sugerencia de creación de una Agencia Europea de Inteligencia que integrara la Inteligencia estratégica de la Unión ha quedado finalmente descartada, aunque la necesidad de establecer unas directrices comunes en este ámbito en el seno de la Unión es un hecho innegable, a la vez que imprescindible.

Por ello, la Unión mantiene órganos tanto en el ámbito policial y estratégico como en el militar, que realizan funciones de integración y análisis de la información.

En cuanto a las materias e intereses comunes, la creación de Europol $^{17}$ en 1995 a través del Convenio del mismo nombre, establece un punto de partida en la cooperación entre Estados. El procedimiento de actuación de Europol es el de facilitar el intercambio de información, de conformidad con la legislación nacional de cada Estado miembro, a través de los oficiales de enlace. Estos funcionarios están acreditados por cada país miembro como representantes de sus organismos nacionales. Asimismo, se llevan a cabo análisis para apoyar a cada Estado, a través de la elaboración de informes estratégicos sobre la base suministrada por los países miembros, generada por Europol u obtenida a través de otras fuentes.

Con el fin de cumplir sus funciones, Europol gestiona un sistema de información (Europol Computer System) ${ }^{18}$, directamente alimentado por los Estados miembros, siendo accesible por estos, mediante consulta, a las unidades nacionales, a los oficiales de enlace, al director, a los directores adjuntos, así como al personal que esté debidamente habilitado.

Hay que decir que uno de los inconvenientes que ostenta Europol es la incapacidad para efectuar detenciones, ya que el objetivo principal, no

17 Convención de Europol. http://www.europol.eu.int.

18 El sistema informático de Europol está compuesto por un Sistema de Índice, un sistema de Información: Europol Information System (EIS), y un sistema de análisis: Europol Analysis System (OASIS). 
es el poder coercitivo ejecutivo, sino la provisión de análisis de la información, a través de fuentes abiertas y confidenciales que le suministran las Fuerzas y Cuerpos de Seguridad de los Estados de la Unión Europea.

En cuanto a los informes, hay que decir que son de dos tipos. Por un lado, informes de situación y tendencias, que se realizan anualmente, de octubre a octubre, y son prospectivos. Y por otro lado, el informe-evaluación de la amenaza, en el cuál no solamente se establece previsión, sino también unos parámetros de actuación bajo el estudio de la motivación y las capacidades, como dos de las variables que definen a las amenazas. Este segundo tipo de informe se realiza de forma coyuntural, no siguiendo unas reglas en cuanto a su periodicidad.

Tras el 11-S, en la Unión también se establecieron una serie de medidas para reforzar la coordinación en materia contraterrorista. Un ejemplo de ello es la creación del Grupo sobre Contraterrorismo, formado por los Estados miembros de la Unión, junto con Noruega y Suiza. El objetivo principal es el establecimiento de una cooperación operacional con respecto a la obtención de información y la prevención del terrorismo. Las reuniones se establecen cada tres meses bajo la supervisión del responsable de la UE sobre asuntos de contraterrorismo.

Como se mencionó anteriormente, la creación de órganos de cooperación en la UE en el ámbito policial y de seguridad, no es lo único que se ha establecido para mantener una colaboración eficaz en el seno de la Unión.

En lo que corresponde a la Política Europea de Seguridad y Defensa, la necesidad de establecer una estructura orgánica en el intercambio de Inteligencia militar, era una cuestión fundamental a la hora de realizar las operaciones de gestión de crisis y prevención de conflictos encomendadas. Para ello, se han creado unas estructuras organizativas adecuadas para la obtención, análisis y distribución de la Inteligencia.

Se crea la División de Inteligencia junto con el Estado Mayor de la Unión, como pilares principales en la estructura de integración de la Inteligencia militar en la UE. La División de Inteligencia tiene como cometido integrar informes que son enviados desde los Servicios de cada uno de los Estados miembros, distribuyéndolos a través de la estructura de la PESD. La tarea principal de la División de Inteligencia se centra en conocer las regiones en las que ha estallado una crisis o conflicto o en las que son susceptibles de que potencialmente puedan surgir. Además, tiene la misión encomendada de apoyar la planificación estratégica que comienza en cuanto emerge una crisis y termina cuando las autoridades políticas de la Unión aprueban o establecen una inter- 


\section{La Cooperación Internacional entre Servicios}

vención militar ${ }^{19}$. Asimismo, establece valoraciones de situaciones, control estratégico y apoyo operacional. En este órgano, hay al menos un experto de cada Estado miembro y trabajan directamente para el Estado Mayor de la UE, manteniendo los vínculos con los respectivos Servicios de Inteligencia nacionales, recibiendo y/ o requiriendo Inteligencia de los mismos cuando sea necesario.

El Estado Mayor de la UE dentro de la estructura del Consejo es la principal fuente de expertos militares en la Unión. En el transcurso de una crisis, la función de este órgano es la de proporcionar alerta temprana, valoraciones de la situación y planificación estratégica para el desarrollo de las «Operaciones Petersberg», incluyendo, la determinación de las Fuerzas nacionales y multinacionales europeas.

Asimismo, se estableció el Centro de Situación, el cuál une las fuentes de Inteligencia civiles y militares. Es el responsable de proporcionar al Alto representante de la PESC la Inteligencia necesaria para realizar una valoración y control de cualquier situación. En su estructura se estableció una célula para obtener y analizar la Inteligencia en colaboración con los Servicios de Inteligencia nacionales.

No hay que dejar a un lado al Centro de Satélites de Torrejón de Ardoz, el cuál transferido a la UE cuando la UEO fue absorbida, por la primera, este centro es el responsable de además de obtener imagenes por medio de satélites, realiza análisis e integración de IMINT, poniéndolo a disposición de las Instituciones de la UE y de sus estructuras militares.

En cuanto al factor regional, las distintas «Europas» que residen en la Unión hacen que la consecución de un objetivo general resulte difícil. Las percepciones e intereses ante una determinada cuestión que poseen unos países no tienen por qué coincidir con las de otros, por lo que a la hora de llegar un entendimiento, el desequilibrio de poder que se produce hace que no se llegue a la consecución del fin propuesto. En este sentido, y como ejemplo, la visión que los Estados mediterráneos tenemos acerca de la inmigración irregular, es diferente de la que poseen los Estados nórdicos. Por lo tanto, el establecimiento de áreas geográficas de integración de Inteligencia, van a ser hechos lógicos, no sólo a la hora de compartir inquietudes, sino por la proxi midad geográfica como modo de localizar los intercambios de información.

De esta forma, en Europa se estableció la Conferencia de Europa Central como forma de participación de los Servicios de Inteligencia e Infor-

19 B. MüLLER-WILLE, «For our eyes only? Shaping an Intelligence Community with the EU». Occasional Papers, Institute for Security Studies. European Union, n. ${ }^{\circ}$ 50, (Enero 2004). 
mación de los Estados de la Europa central y occidental para establecer un vínculo de cooperación e intercambio de información en la lucha contra el terrorismo.

A nivel internacional, las alianzas regionales de cooperación son una práctica común. Así, se pueden destacar el Centro Contraterrorista del Sudeste Asiático, creado bajo el eje del Foro Regional ASEAN y el Grupo TRIDENT, formado por Israel, Turquía e Irán (hasta la Revolución Islámica) en sus comienzos, estableciéndose en la actualidad con la India, Is rael y Turquía con el objetivo de intercambiar inteligencia y apoyo operacional contra el fenómeno terrorista local ${ }^{20}$.

Por tanto, aunque la cooperación multilateral requiera, ciertamente, de unas directrices claras para su consecución, no es imposible. Lo importante es el establecimiento de unos objetivos claros y unas medidas de creación de confianza entre los Estados.

\section{Conclusiones}

Se ha comprobado que las actuaciones de los Servicios de Inteligencia e información siguen las directrices de las políticas estatales en cuanto a sus intereses y percepciones. Siendo así, la voluntad de cooperar con otros Estados resulta complicado puesto que ningún país está dispuesto a ceder información que pueda serle útil para la salvaguarda de sus intereses nacionales.

Con ello, no se quiere decir que la cooperación no pueda llevarse a cabo, sino todo lo contrario. La cuestión radica en conocer los mecanismos para efectuarla. Por tanto, para que la cooperación en materia de Inteligencia resulte efectiva tiene que establecerse equilibrios de poder entre los Estados. Si las ganancias relativas conseguidas por cada Estado en el proceso de cooperación de Inteligencia no producen a largo plazo una mejora evidente de la posición relativa de unos sobre otros, ni se plantea este tipo de percepción, entonces sí se puede producir una cooperación efectiva. De esta forma, la consecución de acuerdos entre las distintas voluntades resultará más eficaz, no viéndose perjudicados los intereses particulares.

${ }^{20}$ M. RUDNER, «Hunters and Gatherers: The Intelligence Coalition Against Islamic Terrorism». op. cit. 